\title{
The temperature dependence of the pion form factor
}

\author{
Jan van der Heide* \\ Fakultät für Physik, Universität Bielefeld, D33615 Bielefeld, Germany \\ E-mail: jan@physik.uni-bielefeld.de
}

Justus H. Koch

National Institute for Nuclear Physics and High-Energy Physics (NIKHEF), 1009 DB

Amsterdam, The Netherlands

and

Institute for Theoretical Physics, University of Amsterdam, Valckenierstraat 65, 1018 XE

Amsterdam, The Netherlands

E-mail: justus@nikhef.nl

\section{Edwin Laermann}

Fakultät für Physik, Universität Bielefeld, D33615 Bielefeld, Germany

E-mail: edwin@physik.uni-bielefeld.de

\begin{abstract}
We calculate the electromagnetic form factor of the pion in quenched lattice QCD. The nonperturbatively improved Sheikoleslami-Wohlert lattice action together with the $\mathscr{O}(a)$ improved current are used to reduce discretisation effects. We obtain form factors for pion masses as low as $m_{\pi}=360 \mathrm{MeV}$. From the low $Q^{2}$ behaviour, we extract the pion radius and use (quenched) chiral perturbation theory to extrapolate the result towards the physical pion mass. In addition, we present the first finite temperature lattice results for the matrix element of the electromagnetic current between pion states and discuss possible differences to the zero temperature case.
\end{abstract}

XXIIIrd International Symposium on Lattice Field Theory

25-30 July 2005

Trinity College, Dublin, Ireland

\footnotetext{
* Speaker.
} 


\section{Introduction}

The pion, being the 'simplest' particle in the hadronic spectrum has been studied intensively on the lattice. Especially the electro-magnetic form factor has received quite some attention. The pioneering work was done by Martinelli and Sachrajda [1], followed by a more detailed study of Draper et al. [2]. More recently, these calculations were extended to improved actions [3,4], and the inclusion of dynamical quarks (see e.g. [5]).

Present and planned experiments at SPS, RHIC, LHC and FAIR attempt to create and explore QCD close to or in its deconfined regime. In this context, it is interesting to know whether and how the charge distribution of hadronic states is subject to changes within such a medium. To investigate this, we have extended the above mentioned results, and calculated the electro-magnetic interaction of a photon and a pion at finite temperature below as well as above the anticipated phasetransition of QCD, for the first time using lattice QCD.

\section{The method}

In our calculations we make use of non-perturbatively improved, quenched Wilson fermions on a $24^{3} \times 32$ lattice $(T \approx 0)$, and on $32^{3} \times 8,6$, and 4 lattices $\left(T \approx 0.93,1.23\right.$ and $\left.1.86 T_{c}\right)$. The gauge coupling, $\beta=6.0$, corresponds to a lattice spacing $a=0.105 \mathrm{fm}$. We generated $\mathscr{O}(100)-\mathscr{O}(200)$ configurations. We calculated correlation functions for five different quark masses below $T_{c}$ and two, including $m_{q}=0$, above $T_{c}$. Details of our methods, including analysis techniques, can be found in [3]. Here, we will only focus on the results and the details of the finite temperature calculations.

In contrast to the $T=0$ case, for which we can write the pion-photon matrix element as

$$
\Gamma_{\mu}^{(T=0)}=\left\langle\pi\left(p_{\mathrm{f}}\right)\left|\gamma_{\mu}\right| \pi\left(p_{\mathrm{i}}\right)\right\rangle=\left(p_{\mathrm{f}}+p_{\mathrm{i}}\right)_{\mu} F\left(Q^{2}\right),
$$

the parametrisation of the matrix element is more involved at finite temperature due to the introduction of a heat bath. Here, the matrix element now involves three form factors and they might dependent on more scalar variables

$$
\Gamma_{\mu}=\left(p_{\mathrm{f}}+p_{\mathrm{i}}\right)_{\mu} F\left(Q^{2}, \mathbf{p}_{\mathrm{f}}^{2}, \mathbf{p}_{\mathrm{i}}^{2}\right)+q_{\mu} G\left(Q^{2}, \mathbf{p}_{\mathrm{f}}^{2}, \mathbf{p}_{\mathrm{i}}^{2}\right)+n_{\mu} H\left(Q^{2}, \mathbf{p}_{\mathrm{f}}^{2}, \mathbf{p}_{\mathrm{i}}^{2}\right),
$$

where $n_{\mu}$ is four velocity of the aforemmentioned heat bath. Since the temperature is defined through the inverse of the temporal extension, higher temperatures are reached for smaller $N_{\tau}$. This means that the two- and three-point functions cannot be measured for large $t$, as one usually does. The correlation functions are thus contaminated with excited states to such an extent that a reliable extraction of the form factor becomes impossible. We therefore turn to spatial correlation functions.

The above mentioned decomposition then changes slightly and the dependence on the scalar variables becomes

$$
\Gamma_{\mu}=\left(p_{\mathrm{f}}+p_{\mathrm{i}}\right)_{\mu} F\left(Q^{2}, \omega_{n, \mathrm{f}}, \omega_{n, \mathrm{i}}\right)+q_{\mu} G\left(Q^{2}, \omega_{n, \mathrm{f}}, \omega_{n, \mathrm{i}}\right)+n_{\mu} H\left(Q^{2}, \omega_{n, \mathrm{f}}, \omega_{n, \mathrm{i}}\right) .
$$

with $\omega_{n, \mathrm{i}}$ and $\omega_{n, \mathrm{f}}$ the Matsubara frequencies of the initial and final states. Please note that due to spherical symmetry of the self-energy, a dependence on the perpendicular momenta $\left(\mathbf{p}_{\perp}=\left(p_{x}, p_{y}\right)\right)$ 


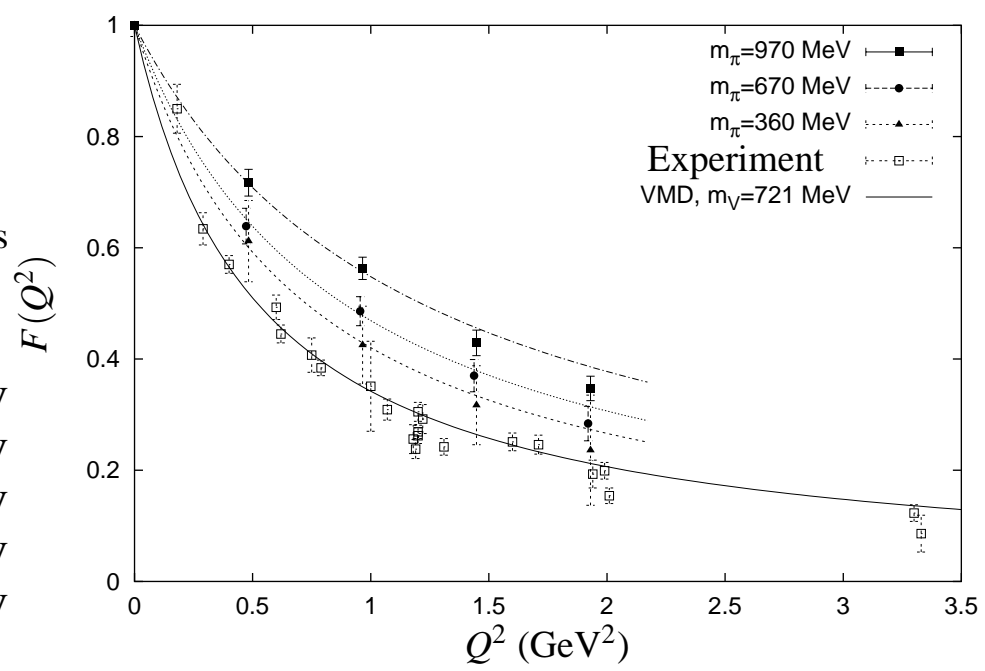

Figure 1: The form factor at $T=0$ for different $m_{\pi}$, compared to experiment $[6,7]$. The curves are VMD fits.

is not allowed. Using current conservation $\left(q_{\mu} \Gamma_{\mu}=0\right)$, we can remove one form factor. Imposing the extra kinematical restrictions

$$
\omega_{n, \mathrm{f}}=\omega_{n, \mathrm{i}}=0
$$

and choosing $\mu=3$, we are left with a parametrisation of the matrix element which only contains $F$ as in the zero temperature case. In the following, we will therefore call $F$ the form factor.

A point of care concerns the renormalisation of the (improved) current. Although the improvement and renormalisation constants should be independent of the temperature, the latter of them can, however, depend on the correlation direction.

\section{Results}

\section{1 $T=0$}

As a byproduct of the calculation of the two-point function, we obtain pion masses for the 5 different $\kappa$-values. They agree with the literature. We also checked the energy-momentum relation and up to the energies involved we found that a continuum relation provides the best description.

Using different currents, we extract the form factor for the five $\kappa$-values at $T=0$. Comparison of the results for the Noether current and the improved current shows that the effect of improvement can be as large as $25 \%$ for the highest momentum transfers considered. The improved results are shown in Fig.1. The high accuracy of the data point at $Q^{2}=0$ is due to the fact that the Ward-Takahashi identity related to current conservation is satisfied to $1 \mathrm{ppm}$. From the figure, we observe that our results approach the experimental data when the quark mass is lowered. Although our lightest pion is still more than twice as heavy as the physical pion, we come rather close to the measurements. As in the previous study [2] of the pion form factor we have compared our results to a VMD inspired monopole form

$$
F\left(Q^{2}\right)=\frac{1}{1-Q^{2} / m_{V}} .
$$




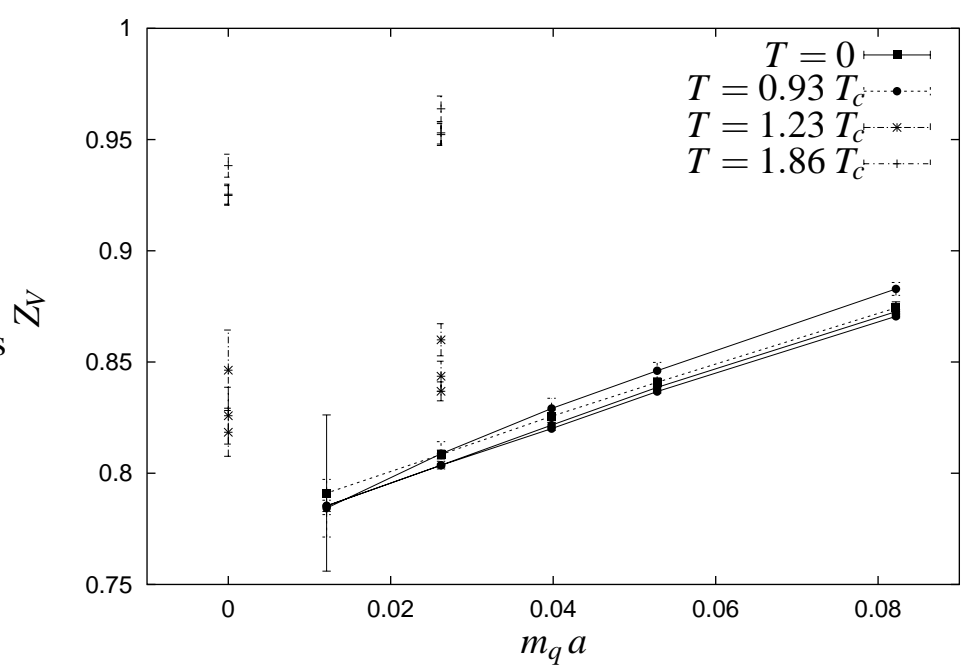

Figure 2: The renormalisation constant $Z_{V}$ for different temperatures and different pion momenta. $Z_{V}$ increases slightly with $p_{\perp}$.

Fitting our data to this model, we extract a vector meson mass, $m_{V}$, which is within $5 \%$ of the corresponding rho mass on the lattice [8]. For completeness, we have also fitted the experimental values (solid line). From the model we can also extract estimates for the pion radius according to

$$
\left\langle r^{2}\right\rangle=-\left.6 \frac{\partial F}{\partial Q^{2}}\right|_{Q^{2}=0}=\frac{6}{m_{V}^{2}},
$$

which are shown in Fig. ๑. For more details, please see [3].

\section{$3.2 T \neq 0$}

The screening masses we extract from the two-point function at $T=0.93 T_{c}$ are equal to the masses at $T=0$. Again, the data for finite momentum is best described by the continuum dispersion relation. The pion masses at $1.86 T_{c}$ are independent of the quark mass and amount to $0.85 \times 2 \omega_{1}$. At the intermediate temperature, the pion screening mass lies between the zero temperature results and $2 \omega_{1}$. Results for $m_{q}=0$ at this temperature were not reliable.

The renormalisation constants mentioned at the end of the last paragraph are determined as follows. First, we have established that the conserved current, which needs no renormalisation, gives $F(0)=0$. This is done using a second current insertion to ensure that no current is leaking away in the opposite direction. The renormalisation of the (improved) local current is then computed from

$$
Z_{V} \equiv Z_{V}^{0}\left(1+b_{V} m_{q}\right)=G_{3}^{c o n}\left(0, p_{\mathrm{f}}, p_{\mathrm{i}}\right) / G_{3}^{l o c}\left(0, p_{\mathrm{f}}, p_{\mathrm{i}}\right)
$$

The influence of the correlation direction can be considerable, as can be seen from Fig. 2. Apart from the renormalisation constants, we continue to use the improvement constant $c_{V}$ as determined in [9].

The form factor at $0.93 T_{c}$ is equal to the zero temperature data. However a slight dependence on the pion momenta has been observed. This is most probably a statistical fluctuation. We combine the lower momenta data in order to facilitate the VMD fit. The data for all temperatures are 


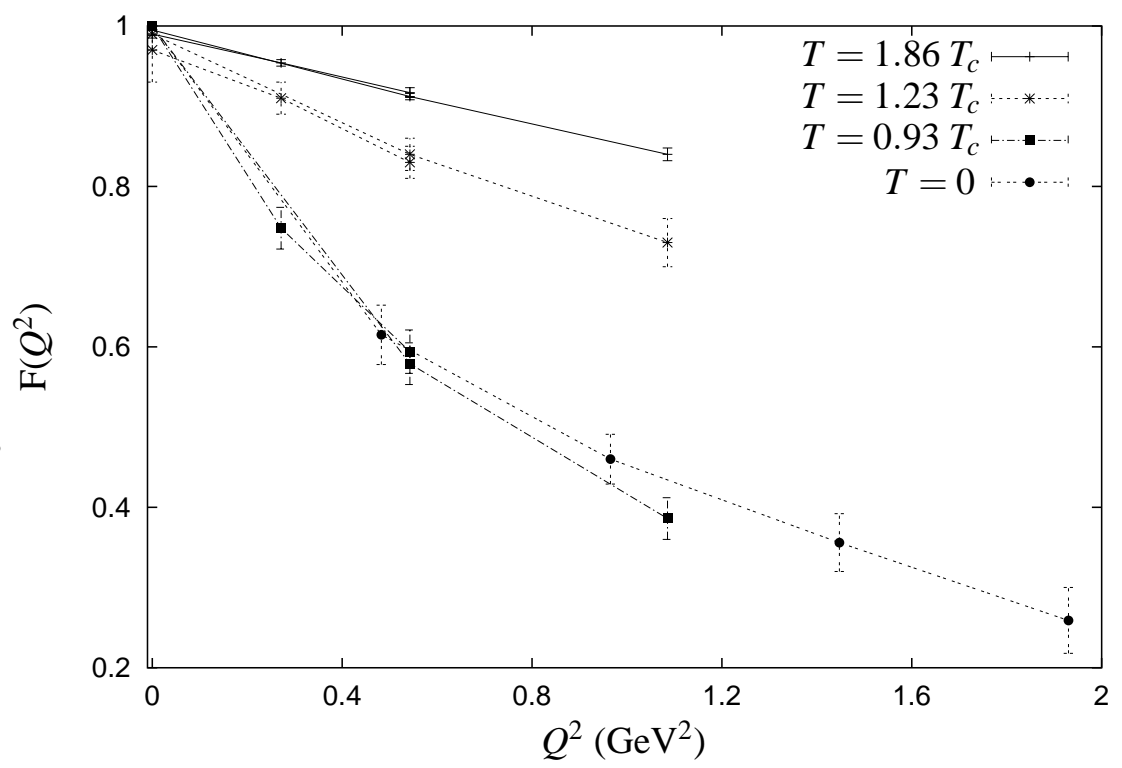

Figure 3: Form factor for different temperatures and a $\kappa$-value corresponding to $m_{\pi}(T=0)=540 \mathrm{MeV}$.

summarised in Fig. 3. The dependence on the transverse pion momenta is not observed for temperatures above $T_{c}$. As for the zero temperature case, the form factor data, below as well as above $T_{c}$, can be described by the VMD inspired monopole form with $m_{V}$ acting as a free parameter up to moderate $Q^{2}$. From a fit to this model we extract the radii, shown in Fig. 6 . Here, the definition of the radius is adjusted according to using spatial correlators.

\section{Discussion}

We have presented the results of a study on the temperature dependence of the electromagnetic interaction of the pion and the photon. The results were obtained using improvement techniques, rendering the simulation free of order $a$ effects. For $T=0$, the form factor was shown to come close to the experimental values, and a pion radius was found within $10 \%$ of the measured value. A further lowering of the quark mass is most likely not the route to follow, indeed, other groups have performed unquenched simulations [5], whose results also show agreement with experiment.

In the second part, we have presented the first finite temperature calculation of the form factor corresponding to $F$ at $T=0$, using lattice QCD. It was found that up to a temperature close to $T_{c}$, the situation is basically unchanged. We found a small dependence on the transverse pion momenta. This is not allowed and most probably due to low statistics. The effect vanishes for temperatures above the critical one. The data above $T_{c}$ is much less 'steep' than at zero temperature, indicating a more point-like behaviour, or equivalently, a smaller radius. This might be due to the fact that we are forced to use spatial correlators. It is known that the spatial string tension above $T_{c}$ is rising with temperature [10]. This could explain why our 'spatial' radii decrease with temperature, where one would expect the opposite for real pion radii. 


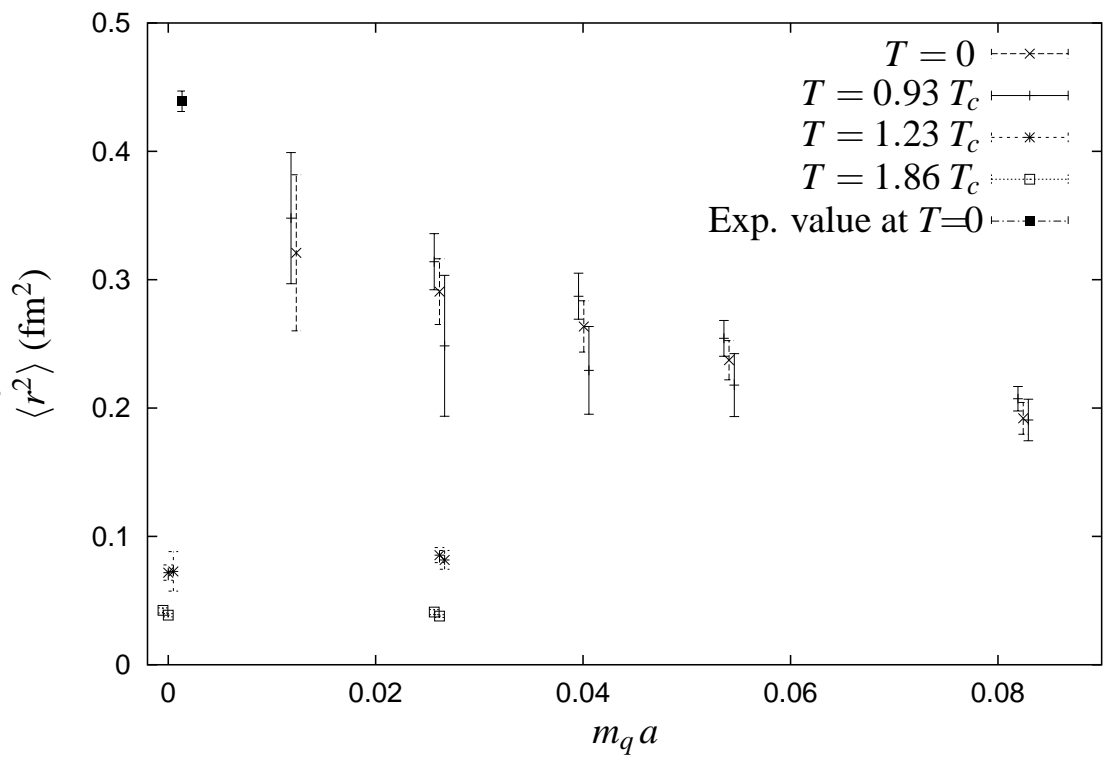

Figure 4: Pion radius for different temperatures and pion momenta. For $T=0.93 T_{c}$, the higher values represent the lower momenta.

\section{References}

[1] G. Martinelli and C. T. Sachrajda, A lattice calculation of the pion's form-factor and structure function, Nucl. Phys. B306 (1988) 865.

[2] T. Draper, R. M. Woloshyn, W. Wilcox, and K.-F. Liu, The pion form-factor in lattice qcd, Nucl. Phys. B318 (1989) 319.

[3] J. van der Heide, J. H. Koch, and E. Laermann, Pion structure from improved lattice qcd: Form factor and charge radius at low masses, Phys. Rev. D69 (2004) 094511, [hep-lat/0312023].

[4] RBC Collaboration, Y. Nemoto, Pion electromagnetic form-factor with domain wall fermions, hep-lat/0309173.

[5] Lattice Hadron Physics Collaboration, F. D. R. Bonnet, R. G. Edwards, G. T. Fleming, R. Lewis, and D. G. Richards, Lattice computations of the pion form factor, hep-lat/0411028.

[6] C. J. Bebek et al., Electroproduction of single pions at low epsilon and a measurement of the pion form-factor up to $q^{* * 2}=10$ - gev **2, Phys. Rev. D17 (1978) 1693.

[7] The Jefferson Lab F(pi) Collaboration, J. Volmer et al., New results for the charged pion electromagnetic form-factor, Phys. Rev. Lett. 86 (2001) 1713-1716, nucl-ex/0010009.

[8] UKQCD Collaboration, K. C. Bowler et al., Quenched qcd with o(a) improvement. $i$ : The spectrum of light hadrons, Phys. Rev. D62 (2000) 054506, [hep-lat/9910022].

[9] T. Bhattacharya, R. Gupta, W.-J. Lee, and S. R. Sharpe, Order a improved renormalization constants, Phys. Rev. D63 (2001) 074505, [hep-lat/0009038].

[10] F. Karsch, E. Laermann, and M. Lutgemeier, Three-dimensional su(3) gauge theory and the spatial string tension of the (3+1)-dimensional finite temperature su(3) gauge theory, Phys. Lett. $\mathbf{B 3 4 6}$ (1995) 94-98, [hep-lat/9411020]. 